PsiCall collect the data of the users, in agreement with RGPD, for descriptive and efficacy studies of the psychological intervention. Teaching: operators recibe specific training in telematic psychological intervention to carry out a better assitance.

Results PsiCall has attended more than 800 students who present psychological and social emergencies since 2017.

Conclusion PsiCall is a pioneering telematic psychological assitance service in Spanish universities that contributes to improve student's wellness at the Complutense University of Madrid.

Conflict of interest None.

Funding Universidad Complutense de Madrid.

\section{PATIENT OUTCOME IN THE DANISH QUALITY DATABASE FOR PREHOSPITAL EMERGENCY MEDICAL SERVICES}

${ }^{1} \mathrm{HC}$ Christensen*, ${ }^{2} \mathrm{EF}$ Christensen, ${ }^{3} \mathrm{PA}$ Berlac. ${ }^{1} T h e$ Danish Clinical Registries (RKKP), Denmark; ${ }^{2}$ Aalborg University, Denmark; ${ }^{3}$ Emergency Medical Services Copenhagen, Denmark

\subsection{6/bmjopen-2019-EMS.26}

Background A national Prehospital Quality Database ${ }^{1}$ has been established in Denmark, and most quality indicators reported are reflecting the administration of the service. In the effort to change the perspective of the register toward patient-centered care and outcomes, cardiac arrest data has been sought from the national digital patient chart used by all medical personal in the EMS. The gold standard for comparison was The Danish Cardiac Arrest Register based on paper reports.

Method The study population consists of all emergency patients, where the entrance to health care is a 1-1-2 call forwarded to one of the five regional emergency medical coordination centers in Denmark (300,000-3 50000 annually). The use of medical data on patients treated with cardiac arrest was collected directly from the medical charts to compare level of registration and quality of treatment. These data were available for 2016 and 2017.

Results From the medical charts we extracted 2576 patients in 2017 with witness cardiac arrest with $28.0 \% \quad(n=$ ?) had ROSC. Among patients receiving CPR 48.6\% $(n=)$ achieved ROSC. Correspondingly in $25.1 \%$ and $47.5 \%$ in 2016. In 2016 DHR reported an incidence of 66 per 100000 inhabitants, where the new data yields 85 per 100000 . This suggests an underreporting to the register during the time of paper reports.

Conclusion National monitoring of health quality should reflect patient outcome. Extracting data directly from the medical charts is not without challenges but is feasible and will help to raise the standard of care.

\section{REFERENCE}

1. Christensen-EF, Berlac-PA, Nielsen-H, Christiansen-CF: The Danish quality database for prehospital emergency medical services. Clinical Epidemiology 2016:8 667-671.

Conflict of interest None.

Funding None.
27 PREHOSPITAL STROKE CODE. EXPERIENCE IN AN ARGENTINEAN EMERGENCY MEDICAL SERVICE

${ }^{1} \mathrm{~A}$ Apesteguía, ${ }^{1} \mathrm{~A}$ Savia*, ${ }^{1} \mathrm{~A}$ Farías, ${ }^{2} \mathrm{~A}$ Muller, ${ }^{2} \mathrm{M}$ Kamijo, ${ }^{2} \mathrm{C}$ Yaryour, ${ }^{2} \mathrm{E}$ Sorkin, ${ }^{2} \mathrm{M}$ Allende. 'ACUDIR Emergencias Médicas, Buenos Aires, Argentina; ${ }^{2}$ OSDE Obra Social de Ejecutivos y Personal de Dirección de Empresas, Argentina

\subsection{6/bmjopen-2019-EMS.27}

Background Stroke is a time dependent medical emergency ${ }^{1}$ that requires the coordinated action of the pre and in-hospital teams to improve the results. ${ }^{2}$ We present the epidemiological characteristics of the population requesting medical attention to a private EMS identified by the telephone operator with activation of a Prehospital Stroke Code (PSC).

Method Observational, retrospective, cross-sectional and descriptive study based on records made through the App Stroke Code v1.0 (internal development) from 01/09/2018 to $31 / 1 / 2019$. $n=97$ cases. The data was transferred to a Microsoft Excel spreadsheet and to Epi Info v7.2.2.1 for analysis.

Results PSC was activated in 97 cases. 58 has a suspected Stroke and in 39, the code was canceled by on-scene-EMS team due alternative prehospital diagnosis. The mean age was 78 y/o. 44patients (76\%) consulted within three hours of the onset of symptoms. CINCINATTI-prehospital scale average on arrival was 2 points. $100 \%$ of patients with CINCITATTI $0-1$ consulted after 3 hs (15) (p0.03). On patients with CINCINATTI-score of 3,97\% (p.0038) were hypertensive at the time of the consultation. No hypoglycaemia was found. Of the 58 suspected stroke cases, 16 received some form of reperfusion treatment (rTPA IV, thrombectomy-intraarterial rTPA or combination). The remainder 43 patients who did not receive reperfusion treatment were mainly due to: Alternative in-hospital diagnosis, out of therapeutic window and Intracranial bleeding. The average door to needle time was 37 minutes.

Conclusion Patients more symptomatic consulted earlier 27\% of the patients received reperfusion therapy, dramatically surpassing the average of our country $(1 \%$ according to the RENACER registry3).

\section{REFERENCES}

1. Saver JL. Time Is Brain - Quantified. Stroke 2006;37:263-266.

2. Caputo LM, Jensen J, Whaley M, et. al. How a CT-Direct Protocol at an American Comprehensive Stroke Center Led to Door-to-Needle Times Less Than 30 Minutes. The Neurohospitalist 2017, Vol. 7(2) 70-73.

3. Sposato LA, Esnaola MM, Zamora $R$ and the ReNACer Investigators. Quality of ischemic stroke care in emerging countries: the Argentinian National Stroke Registry (ReNACer). Stroke. 2008 Nov;39(11):3036-41.

Conflict of interest None.

Funding None.

\section{PAIN MANAGEMENT OF PEDIATRIC TRAUMA PATIENT IN A PREHOSPITAL MEDICAL SERVICE}

SI Montero*, CL Villamor, JA Lara, T Casado, PJ Uriarte, F Miguel. SAMUR Civil Protection, Emergency Medical Service, Madrid, Spain

\subsection{6/bmjopen-2019-EMS.28}

Background Pain produced by trauma events is one of the most common problems of prehospital settings. However, pain control in children continues being a challenge for health 